\title{
Entropy
}

ISSN 1099-4300

www.mdpi.org/entropy/

\section{ENTROPY GENERATION IN PRESSURE GRADIENT ASSISTED COUETTE FLOW WITH DIFFERENT THERMAL BOUNDARY CONDITIONS}

\author{
Abdul Aziz \\ Department of Mechanical Engineering, Gonzaga University, Spokane, WA 99258, USA. \\ Email:aziz@gonzaga.edu
}

Received: 9 February 2006 / Accepted: 3 May 2006 / Published: 4 May , 2006

\begin{abstract}
The present paper is concerned with an analytical study of entropy generation in viscous, incompressible Couette flow between a stationary plate and a moving plate. The flow induced by the moving plate is assisted by a constant pressure gradient along the flow direction. Four different combinations of thermal boundary conditions are investigated: (a) plates at different temperatures, (b) stationary plate at a fixed temperature and moving plate subjected to a constant heat flux, (c) stationary plate at a fixed temperature and convection at the moving plate, and (d) convection at both plates.

Besides the velocity and temperature profiles, dimensionless results are presented for the entropy generated due to heat transfer, the entropy generated due to viscous dissipation, and the total entropy generation. These results illustrate the effect of pressure gradient, temperature asymmetry, heat flux, convection Biot numbers, and ambient temperatures. For certain combinations of thermal variables, the total entropy generated is minimized.
\end{abstract}

\section{Keywords:}

Entropy, Couette flow, pressure gradient, thermal boundary conditions.

\section{Introduction}

Since the pioneering work of Bejan [1] on entropy generation in convective heat transfer, it is now widely recognized that convective heat transfer problems that were previously studied using the first law of thermodynamics be reexamined in the light of the second law of thermodynamics so that 
thermal systems can be designed with the objective of minimizing thermodynamic irreversibility. This design methodology, known as entropy generation minimization (EGM), is comprehensively covered in the book by Bejan [2]. The popularity of EGM can be gauged from the number of papers on entropy generation in convective flows that continue to appear in the heat transfer literature e.g. [3, 4]. We will refer only to the literature that deals with entropy generation for flow between parallel plates which is of immediate relevance to the present study.

The flow of a viscous, incompressible flow induced by a plate moving parallel to a stationary plate and assisted by a favorable pressure gradient situation, known as generalized Couette flow,is useful in many engineering applications and has been studied extensively both from fluid mechanics and heat transfer perspectives. However, studies of entropy generation in such flows have been limited. Ibanez et al [5] have studied the entropy generation due to pressure gradient assisted viscous flow between two parallel stationary plates when the plates are subjected to convective boundary conditions. Their analysis assumes that the convective environment temperature is the same for both plates but the heat transfer coefficients and consequently the Biot numbers are different. They concluded that a minimum volumetric entropy generation rate can be achieved for certain combinations of Biot numbers. This work was later extended to the generalized Couette flow [6].

The present paper is an analytical study of entropy generation in generalized Couette flow with focus on the effect of thermal boundary conditions imposed on the plates. Four different combinations of thermal boundary conditions are investigated: (a) both plates at different temperatures, (b) stationary plate at a fixed temperature and moving plate subjected to a constant heat flux, (c) stationary plate at a fixed temperature and convection at the moving plate, and (d) convection at both plates. The case (d) is a refinement of the analysis presented in [6] because it allows both the environment temperatures and the convective heat transfer coefficients to be different. Analytical results for the velocity and temperature profiles, entropy generation due to heat transfer, entropy generation due to viscous dissipation, and the total entropy generation are presented and discussed.

\section{Hydrodynamic and Thermal Analysis}

Fig. 1 illustrates the Couette flow of a viscous, incompressible fluid confined between two parallel plates separated by a distance a. The fluid viscosity, $\mu$, is assumed to be a constant. The bottom plate is stationary while the top plate moves with a uniform $\mathrm{V}$. The fluid flow is also assisted by a favorable pressure gradient, $\mathrm{dp} / \mathrm{dx}<0$.

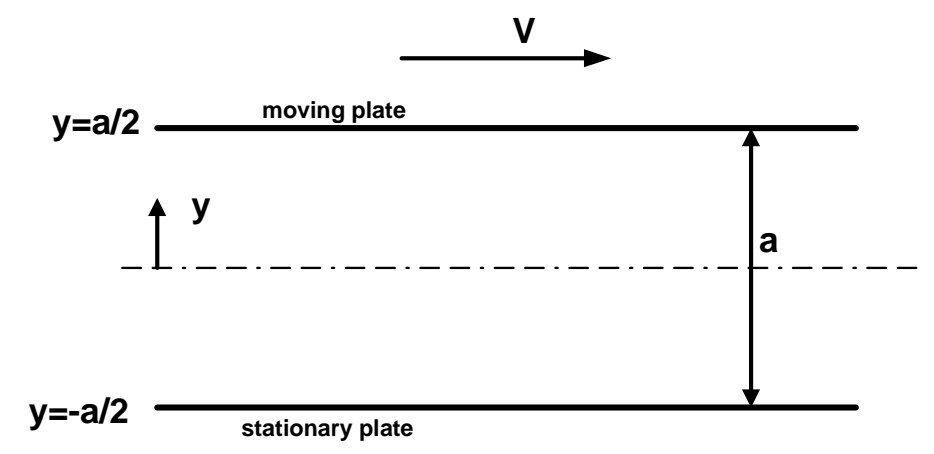

Fig.1. Couette flow between parallel plates 
The momentum equation for the velocity $\mathrm{u}$ along the $\mathrm{x}$ direction may be written in dimensionless form as

$$
\frac{d^{2} U}{d Y^{2}}+P=0
$$

where $\mathrm{U}=\mathrm{u} / \mathrm{V}, \mathrm{Y}=\mathrm{y} / \mathrm{a}$, and $P=-\frac{a^{2}}{\mu V} \frac{d p}{d x}$ is the dimensionless pressure gradient.

The boundary conditions for equation (1) are

$$
\begin{aligned}
& Y=-1 / 2, U=0 \\
& Y=1 / 2, U=1
\end{aligned}
$$

The solution of equations (1-2) is easily obtained as

$$
U=\frac{1}{2}+Y+\frac{1}{8} P\left(1-4 Y^{2}\right)
$$

The derivative of $U$ with respect to $Y$ which will be needed later in the entropy generation analysis is

$$
\frac{d U}{d Y}=1-P Y
$$

With the inclusion of heat conduction in the y direction and the viscous dissipation term, the energy equation in dimensionless form is expressible as

$$
\frac{d^{2} \theta}{d Y^{2}}+\left(\frac{d U}{d Y}\right)^{2}=0
$$

where $\theta=k T / \mu V^{2}$ is the dimensionless temperature and $k$ is the thermal conductivity of the fluid. The use of dimensionless temperature in this form eliminates the Eckert number Ec and Prandtl number Pr which appear in traditional analyses. The solution of equation (5) depends on the thermal boundary conditions imposed at the plates. We consider the solutions for four combinations of boundary conditions.

\section{Constant Plates Temperatures}

The stationary plate is assumed to be at a constant temperature $T_{1}$ and the moving plate at a constant temperature $T_{2}$. In terms of $\theta$, the boundary conditions become

$$
\begin{aligned}
& Y=-1 / 2, \theta=\theta_{1} \\
& Y=1 / 2, \theta=\theta_{2}
\end{aligned}
$$

The solution of equations $(5,6)$ is

$$
\theta=\frac{1}{2}\left(\theta_{1}+\theta_{2}\right)+\frac{1}{8}\left(1+\frac{P^{2}}{24}\right)+\left(\theta_{2}-\theta_{1}-\frac{P}{12}\right) Y-\frac{1}{2} Y^{2}+\frac{P}{3} Y^{3}-\frac{P^{2}}{12} Y^{4}
$$

The derivative of equation (7) with respect to $\mathrm{Y}$ which will be needed later in entropy generation analysis is

$$
\frac{d \theta}{d Y}=\theta_{2}-\theta_{1}-\frac{P}{12}-Y+P Y^{2}-\frac{P^{2}}{2} Y^{3}
$$




\section{Constant Temperature at Stationary Plate and Constant Heat flux at the Moving Plate}

In this case, the boundary condition (6b) changes to

$$
Y=1 / 2, \frac{d \theta}{d Y}=Q
$$

where $Q=q^{\prime \prime} a / \mu V^{2}$ is the dimensionless heat flux. $q^{\prime \prime}$ is the heat flux at the moving plate.

The solution of equation (5) subject to equations (6a) and (9) is

$$
\begin{array}{r}
\theta=\frac{5 P^{4}-16 P^{3}+72 P^{2}+16+192 P^{2} \theta_{1}+96 P^{2} Q}{192 P^{2}} \\
+\frac{P^{3}-6 P^{2}+12 P-8+24 P Q}{192 P^{2}} Y-\frac{(P Y-1))^{4}}{12 P^{2}}
\end{array}
$$

\section{Constant Temperature at Stationary Plate and Convection at the Moving Plate}

In this case, the boundary condition at the moving plate may be written as

$$
Y=1 / 2, \frac{d \theta}{d Y}+\operatorname{Bi}\left(\theta-\theta_{a}\right)=0
$$

where in Biot number $B i=h a / k, h$ is the convection heat transfer coefficient and in $\theta_{a}=k T_{a} / \mu V^{2}, T_{a}$ is the convection environment temperature.

The solution of equation (5) subject to equations (6a) and (11) is

$$
\begin{aligned}
\theta=- & \frac{(P Y-1)^{4}}{12 P^{2}}+\frac{\left[P^{3}-2 P^{2}(B i+3)+12 P\left(1-2 B i \theta_{1}+2 B i \theta_{a}\right)-8(B i+1)\right] Y}{24 P(1+B i)} \\
& +\frac{P^{4}(B i+5)-16 P^{3}+24 P^{2}\left(4 B i \theta_{1}+4 B i \theta_{a}+B i+8 \theta_{1}+3\right)+16(1+B i)}{192 P^{2}(B i+1)}
\end{aligned}
$$

\section{Convection at both plates}

The boundary conditions for this case are

$$
\begin{aligned}
& Y=-1 / 2, \frac{d \theta}{d Y}+B i_{1}\left(\theta-\theta_{a, 1}\right)=0 \\
& Y=1 / 2, \frac{d \theta}{d Y}+B i_{2}\left(\theta-\theta_{a, 2}\right)=0
\end{aligned}
$$

where $B i_{1}=h_{1} a / k, B i_{2}=h_{2} a / k, \theta_{a, 1}=k T_{a, 1} / \mu V^{2}, \theta_{a, 2}=k T_{a, 2} / \mu V^{2}$ and $h_{1}, T_{a, 1}$ and $h_{2}, T_{a, 2}$ are the heat transfer coefficients and convection environment temperatures at the stationary and moving plates, respectively.

The solution of equation (5) subject to the boundary conditions (13) and 14) is

$$
\theta=-\frac{1}{12} P^{2} Y^{4}+\frac{1}{3} P Y^{3}-\frac{1}{2} Y^{2}+C_{1} Y+C_{2}
$$


where $C_{1}=\frac{P^{2}\left(B i_{1}+B i_{2}\right)+2 P\left(3 B i_{2}-3 B i_{1}-2 B i_{1} B i_{2}\right)+12\left(B i_{1}+B i_{2}\right)+24 B i_{1} B i_{2}\left(\theta_{a, 2}-\theta_{a, 1}\right)}{24\left(B i_{1}+B i_{1} B i_{2}-B i_{2}\right)}$

$$
\begin{aligned}
C_{2}=\frac{P^{2}\left(5 B i_{1}-5 B i_{2}+B i_{1} B i_{2}-16\right)-16 P\left(B i_{1}+B i_{2}\right)+72\left(B i_{1}-B i_{2}\right)}{192\left(B i_{1}+B i_{1} B i_{2}-B i_{2}\right)} \\
+\frac{B i_{1} B i_{2}\left(4 \theta_{a, 1} 4 \theta_{a, 2}+1\right)+8\left(\left(B i_{1} \theta_{a, 1}-B i_{2} \theta_{a, 2}\right)\right)+3\left(B i_{1}-B i_{2}\right)-8}{8\left(B i_{1}+B i_{1} B i_{2}-B i_{2}\right)}
\end{aligned}
$$

\section{Entropy Generation Rate}

Adapting the general result for the local volumetric entropy generation rate $\left(W / m^{3} K\right)$ provided by Bejan $[1,2]$ to the present convective flow situation, we have

$$
\dot{S}_{g e n}^{\prime \prime \prime}=\frac{k}{T^{2}}\left(\frac{d T}{d y}\right)^{2}+\frac{\mu}{T}\left(\frac{d u}{d y}\right)^{2}=\dot{S}_{g e n, h}^{\prime \prime \prime}+\dot{S}_{g e n, f}^{\prime \prime \prime}
$$

which may be expressed in dimensionless form as

$$
\frac{\dot{S}_{g e n}^{\prime \prime \prime} a^{2}}{k}=\frac{1}{\theta^{2}}\left(\frac{d \theta}{d Y}\right)^{2}+\frac{1}{\theta}\left(\frac{d U}{d Y}\right)^{2}=S_{h}+S_{f}
$$

Both in equations (18) and (19), the first term represents the entropy generation due to heat conduction (subscript $\mathrm{h}$ ) and the second term the entropy generation due to viscous or fluid friction effect (subscript f). Using equation (4) for $d U / d Y$ and the appropriate results (depending on the thermal boundary conditions) for $d \theta / d Y$, we can integrate equation (19) from $Y=-1 / 2$ to $Y=1 / 2$ and obtain the entropy generation rate across the gap between the plates.

\section{Results and Discussion}

The dimensionless velocity distribution given by equation (3) is plotted in Fig. 2 for $P=0$ (red), 2 (green), 4 (yellow), 6 (blue), 8(pink), and 10 (aqua blue). For $P=0$ i.e. no axial pressure gradient, the velocity distribution is linear giving a constant velocity gradient $d U / d Y$ across the gap. As a consequence, the entropy generation due to fluid friction occurs at all locations across the gap. As the pressure gradient $P$ increases, the velocity profiles exhibit maxima indicating zero velocity gradients at certain locations across the gap and consequently zero entropy generation due to friction at those locations. The location of zero entropy generation due to friction moves closer to the center line $Y=0$ as the pressure gradient increases. 


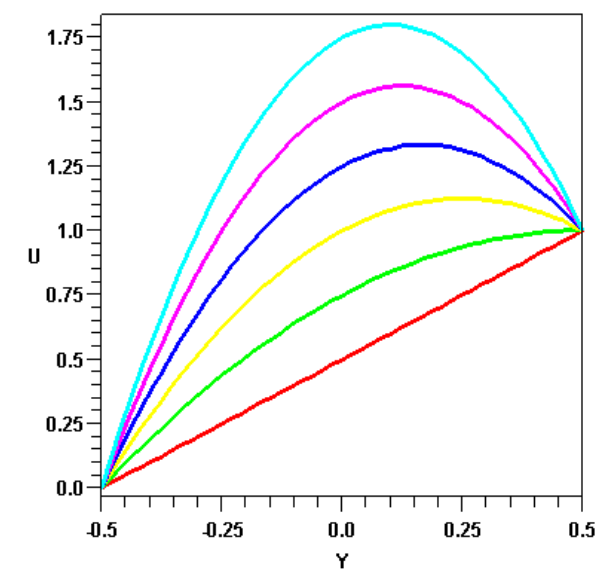

Fig.2. Effect of pressure gradient $\mathrm{P}$ on velocity profiles

\section{Constant Plates Temperatures}

The effect of pressure gradient on the temperature distribution is illustrated in Fig. 3 for the case of constant plate temperatures $\left(\theta_{1}=20, \theta_{2}=5\right)$. In the range from $P=0-10$, the temperature distribution changes only slightly. Since the temperature profiles do not exhibit any maxima, the entropy generation due to heat conduction occurs at all locations across the gap. However, a distinct maximum in temperature is observed at $P=50$ and indicates zero entropy generation due to heat conduction at that location.

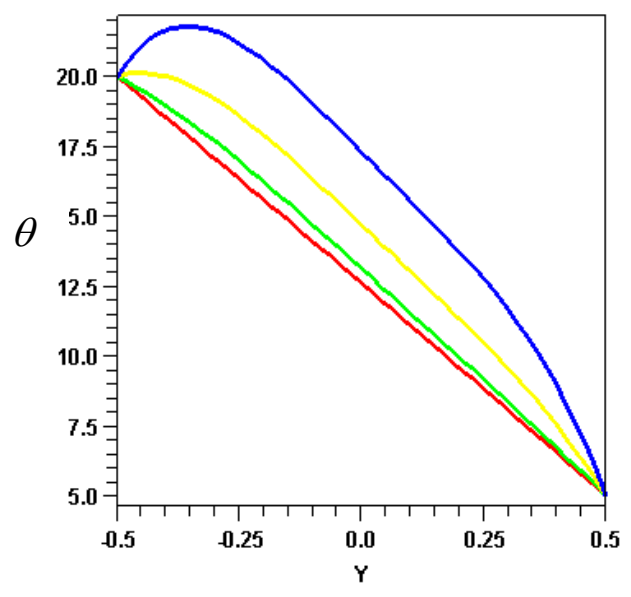

Fig.3. Effect of pressure gradient $\mathrm{P}$ on temperature profile 


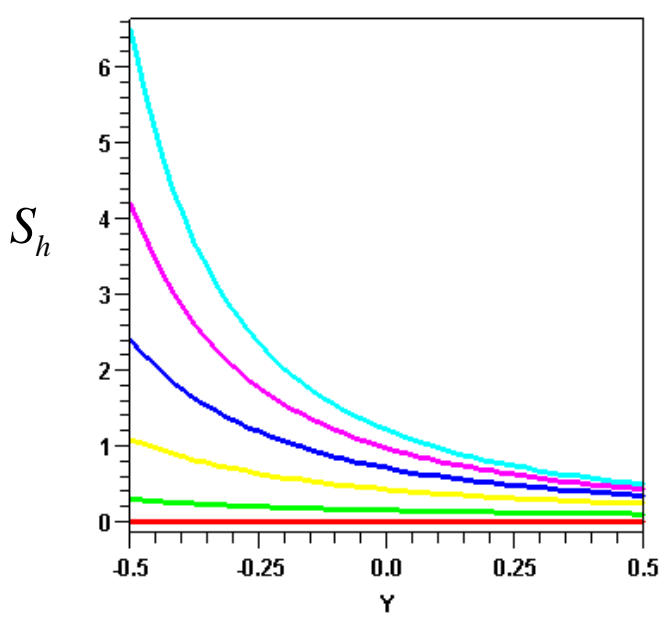

Fig.4a. Local entropy generation due to heat conduction

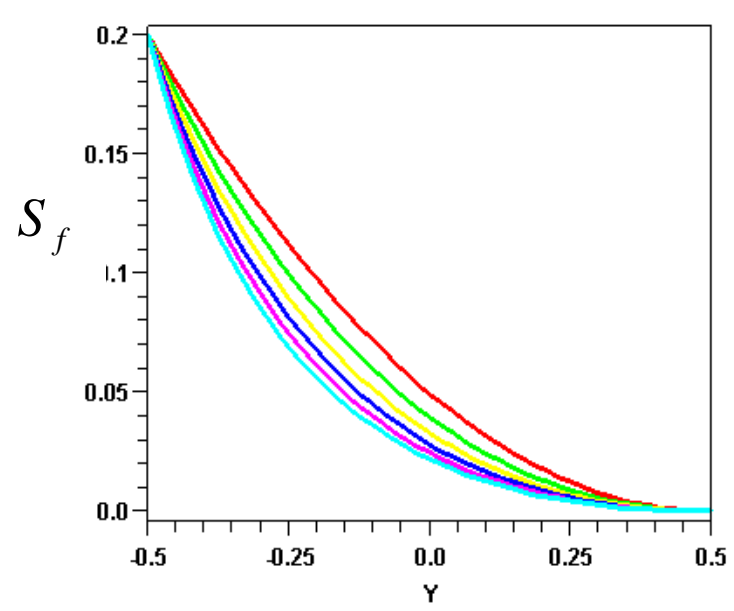

Fig.4b.Local entropy generation due to fluid friction

For fixed values of $\theta_{1}=20$ and $P=2$, the effect of varying $\theta_{2}$ on the entropy generation rate is depicted in Fig.4. Fig. 4a shows the entropy generated due to heat conduction i.e. $\dot{S}_{g e n, h}^{\prime \prime \prime} a^{2} / k\left(\right.$ denoted by $\left.S_{h}\right)$ while Fig. 4 b shows that due to fluid friction i.e. $\dot{S}_{g e n, f}^{\prime \prime \prime} a^{2} / k$ (denoted by $S_{f}$ ). The temperature is varied from $\theta_{2}=20$ (red curve) in increment of 10 to 70 (aqua curve). For $\theta_{2}=20$, the local entropy generation due to heat conduction (Fig 4a) is negligible. However, the entropy generation due heat conduction increases sharply as $\theta_{2}$ is increased particularly at the stationary plate where the largest temperature gradients occur. The effect of $\theta_{2}$ on the local entropy generation at the moving plate is rather small because the changes in $\theta_{2}$ result in comparatively smaller changes in the temperature gradients at the moving plate. The local entropy generation due to fluid friction (Fig. 4b) is maximum at the stationary plate and minimum at the moving plate. The highest entropy generation due to fluid friction occurs at the lowest value of $\theta_{2}$ (red curve). This is due to the presence of $\theta$ in the denominator of the fluid friction term in equation (19). A comparison of the results in Fig. $4 \mathrm{a}$ and $4 \mathrm{~b}$ and the corresponding results for other pressure gradients reveals that the entropy generation due to fluid friction is much smaller than that due to heat conduction for the range of $P$ investigated i.e. from 0 to 50 .

Fig.5 shows the integrated (across the gap) total entropy generation rate, denoted in the figure by $S=S_{h}+S_{f}$ for convenience, as a function of the pressure gradient $P$ for $\theta_{2}=20,40,60$, and 80 with $\theta_{1}$ fixed at a value of 20 . The contribution of $S_{f}$ is much smaller than $S_{h}$. The total entropy generation rate increases as $P$ increases and/or $\theta_{2}$ increases. This is a consequence of the higher velocity and temperature gradients caused by the increase in $P$ and $\theta_{2}$, respectively. From a system design perspective, the total entropy generation is minimized only when the plates are held at identical temperatures and the pressure gradient is zero, that is, when the flow is driven solely by the motion of the upper plate. 


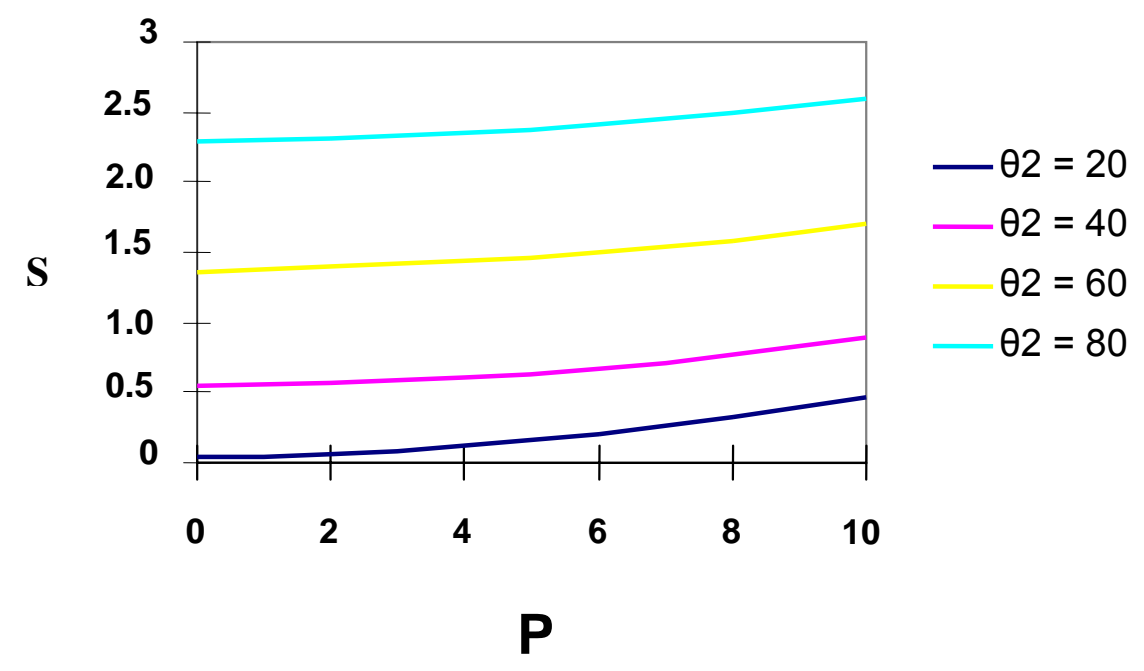

Fig.5. Effect of pressure gradient $\mathrm{P}$ and $\theta_{2}$ on total entropy generation.

\section{Constant Temperature at Stationary Plate and Constant Heat flux at the Moving Plate}

For this case, the temperature at the stationary plate i.e. $\theta_{1}$ was fixed at a value of 20 . The effect of varying the pressure gradient $P$ and the heat flux $Q$ at the moving plate is illustrated in Fig. 6 . The total entropy generation rate increases significantly with increase in pressure gradient but is comparatively less affected by the changes in heat flux at the moving plate. The increase in entropy generation rate is due to the increase in velocity and temperature gradients that accompany the increase in $P$ and $Q$. It may be noted that in this case the moving plate is colder than the stationary plate and extracts energy from the fluid. The entropy generation is minimized only when the moving plate is insulated, the pressure gradient is zero and the flow is induced solely by the motion of the plate. A comparison of Figs.5 and 6 shows that by extracting heat at a constant rate from the moving plate, the entropy generation in the process can be significantly reduced.

For the case where the moving plate is hotter than the stationary plate, the moving plate delivers energy to the fluid. Fig.7 shows the results for this scenario. For low values of pressure gradient $P$, the entropy generation rate increases rapidly as the heat flux increases beyond $Q \approx 8$. It is interesting to observe that at high values of the heat flux, the entropy curves for low values of $P$ cross over the curves for high values of $P$. Thus, within the examined intervals of $P$ and $Q$, the highest entropy generation is encountered at $P=6$ and $Q=16$. However, at higher pressure gradients, the increase in $Q$ affects the entropy generation rate moderately. Furthermore, at $P=18$ and 20, both curves exhibit a minimum in the neighborhood of $Q=8$ (discernible with numerical results). This result is important in flows driven by high pressure gradients in which the moving plate supplies heat to the fluid. If a system is to be designed under these operating conditions, the maximum energy input from the moving plate should be close to $Q=8$ to ensure minimum entropy generation. 


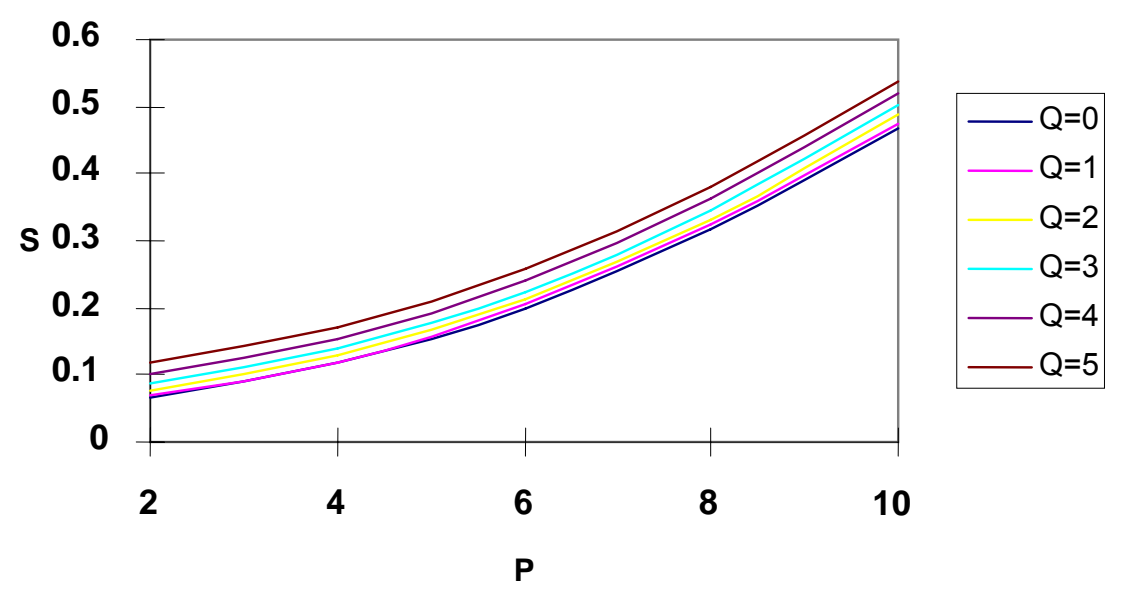

Fig.6. Effect of pressure gradient $\mathrm{P}$ and heat flux $Q$ on total entropy generation when the moving plate delivers heat to the fluid.

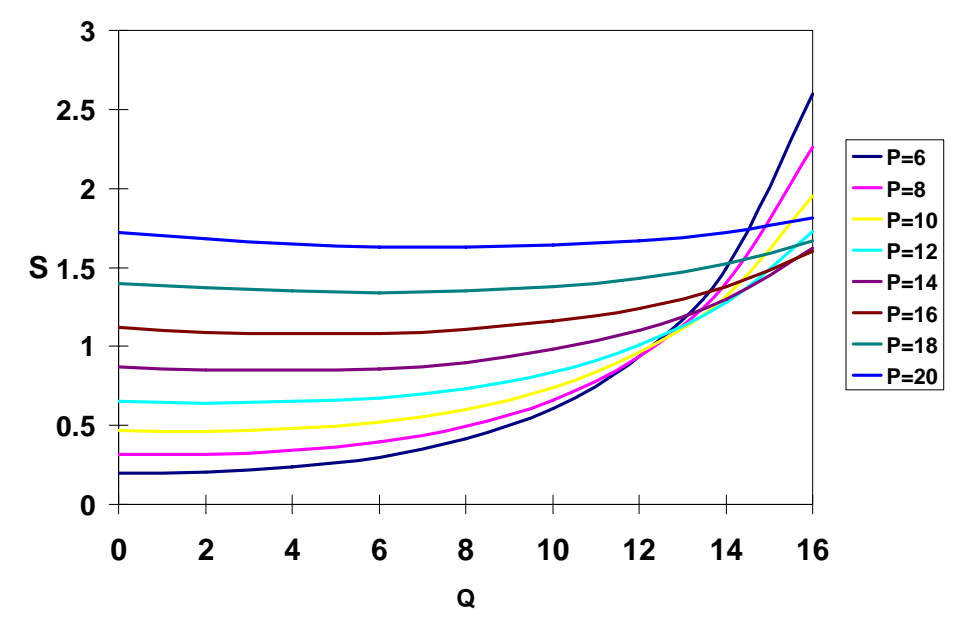

Fig.7. Effect of pressure gradient $P$ and heat flux $Q$ on total entropy generation rate when the moving plate extracts heat from the fluid.

\section{Constant Temperature at the Stationary Plate and Convection at the Moving Plate}

We choose $\theta_{1}=20$ and $\theta_{a}=5$ and illustrate in Fig. 8 the effect of Biot number on the total entropy generation rate for selected values of the pressure gradient. For each value of $P$, the total entropy generation rate increases as $\mathrm{Bi}$ increases i.e. the convection at the moving plate gets stronger. The increase in heat removal from the fluid by the moving plate results in enhanced entropy generation rate. For a fixed $\mathrm{Bi}$, the higher the pressure gradient and hence the larger the velocity gradients, the higher the entropy generation rate. 


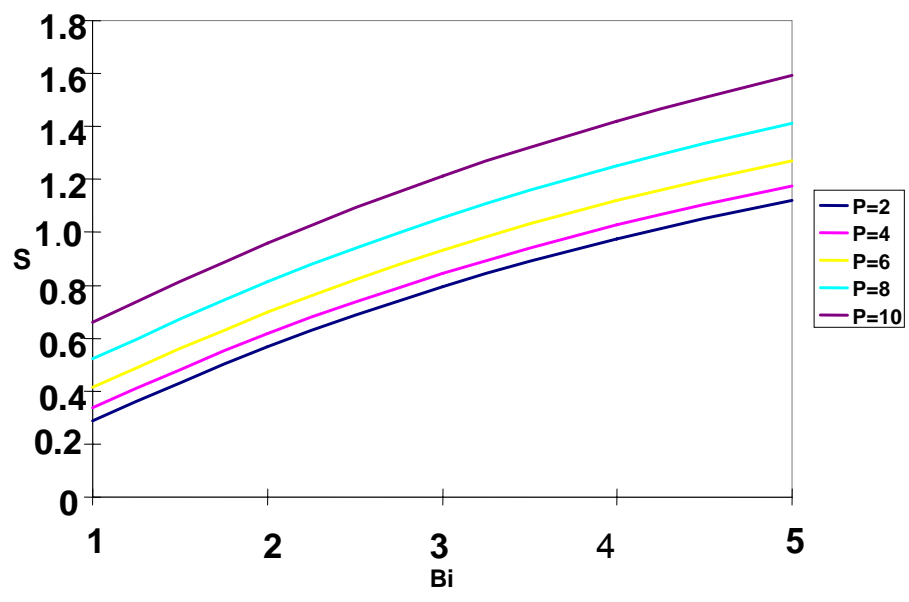

Fig. 8.Variation of entropy generation rate with Biot number Bi and pressure gradient $\mathrm{P}$.

The effect of varying the convection environment temperature $\theta_{a}$ on the entropy generation rate is depicted in Fig. 9 for selected values of the pressure gradient $P$. This figure was generated by fixing $\theta_{1}=20$ and $\mathrm{Bi}=2$. As $\theta_{a}$ increases, the convective heat removal from the moving plate decreases which leads to a reduction in entropy generation rate.

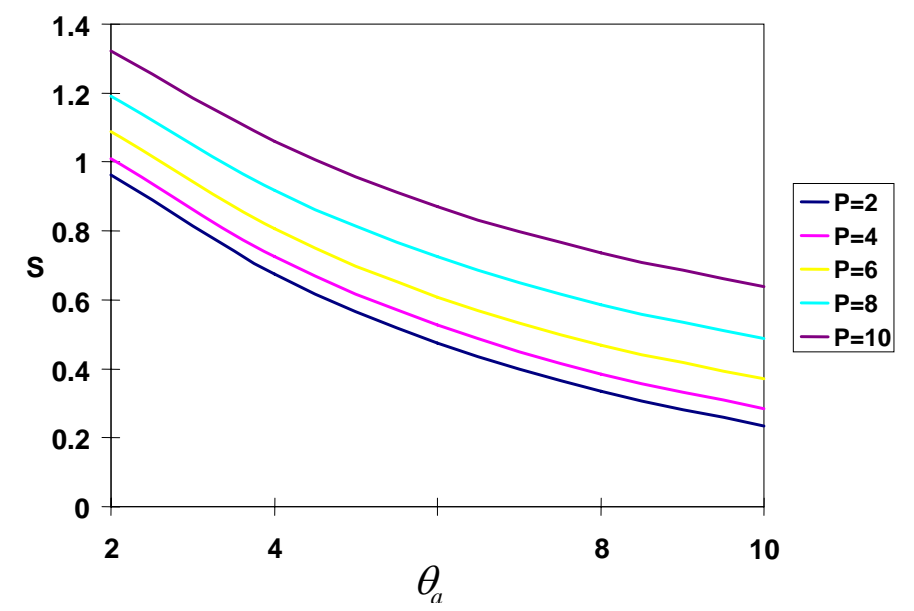

Fig.9. Entropy generation rate as a function of $\theta_{a}$ and $P$ with $\theta_{1}=20$ and $\mathrm{Bi}=2$. 


\section{Convection at both plates}

The variables in this case are $P, \theta_{a, 1}, \theta_{a, 2}, B i_{1}$ and $B i_{2}$. We fix the first three at $P=2, \theta_{a, 1}=20$, $\theta_{a, 2}=10$ and study the effect of varying $B i_{1}$ and $B i_{2}$. In Fig. 10, the entropy generation rate is plotted as a function of $B i_{2}$ for parametric values of $B i_{1}$. At $B i_{1}=1$, the entropy generation increases sharply as $B i_{2}$ increases. However at $B i_{1}=2,3,4$ and 5 , the increase in entropy generation with $B i_{2}$ is moderate. Also the effect of $B i_{1}$ is significantly attenuated beyond $B i_{1}=3$.

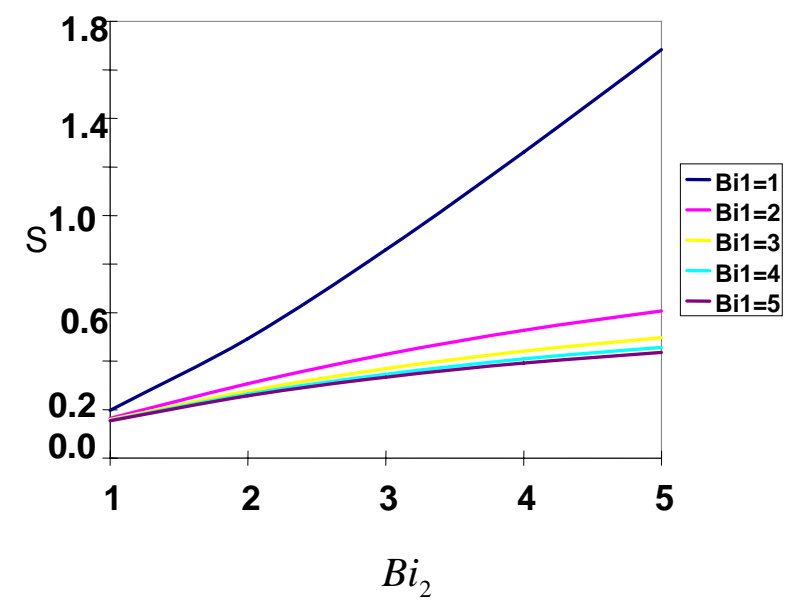

Fig.10. Entropy generation rate as a function of $B i_{2}$ and $B i_{1}$ with $P=2$, $\theta_{a, 1}=20, \theta_{a, 2}=10$.

It has been pointed by Ibanez et al [6] that for certain combinations of $B i_{1}$ and $B i_{2}$, the entropy generation attains a minimum. A close examination of their Fig. 3 for $G=2$ ( $P=2$ in present work) indicates the variation in entropy generation as $B i_{1}\left(B i_{2}\right.$ in present work) increases from 0 to 6 is only about 0.2 percent for the three values of $B i_{2}\left(B i_{1}\right.$ in present work ) used namely 20, 25 and 30 with the minimum entropy generation occurring at $B i_{1}\left(B i_{2}\right.$ in present work $)=0.5$. Because of the variation of 0.2 percent, the minimum could not be identified graphically without a large magnification of $S$ axis. We use the values of $P, \theta_{a, 1}, \theta_{a, 2}, B i_{1}$ and $B i_{2}$ on which their Fig. 3 is based and present our results in Fig.11. It can be observed that in the range of $B i_{2}$ from 0.5-3.0 and $B i_{1} \geq 2$, the entropy generation rate is virtually a minimum. A distinct minimum can be identified by magnifying the $S$ axis or examining the numerical results but the exact determination of the minimum would appear to be of little practical use. The same conclusion was reached with the results for other values of $P, \theta_{a, 1}$, and $\theta_{a, 2}$.

The results of asymmetric convective cooling of the plates provide opportunity for minimum entropy design of Couette flow systems. For minimum entropy generation design, the cooling of the moving plate must provide a minimum Biot number of 2 for the operating condition of $P=2$, 
$\theta_{a, 1}=20, \theta_{a, 2}=10$.The designer then has the flexibility of providing cooling at the stationary plate within Biot numbers in the range 0.5-3.

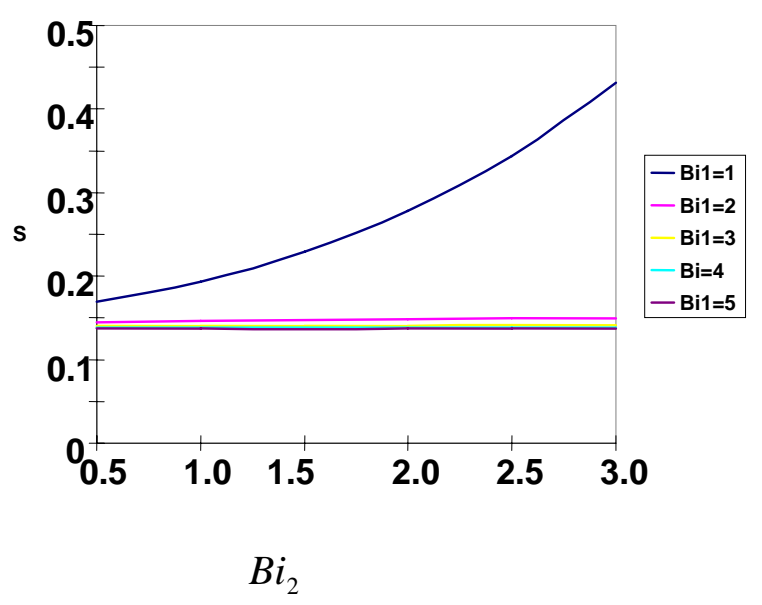

Fig.11. Entropy generation rate as a function of $B i_{2}$ and $B i_{1}$ with $P=2$, $\theta_{a, 1}=10, \theta_{a, 2}=10$.

\section{Conclusions}

An analytical study of entropy generation in a plane Couette flow with favorable pressure gradient and viscous dissipation has been presented for four thermal boundary conditions: (a) constant temperatures at both stationary and moving plates, (b) constant temperature at the stationary plate and a constant heat flux at the moving plate, (c) constant temperature at the stationary plate and convection at the moving plate, and (d) convection at both plates. The effect of pressure gradient and thermal parameters on the entropy generation rate has been presented and discussed in detail. For case (a), minimum entropy design can be realized only when the plates are maintained at identical temperatures and the pressure gradient is zero i.e. the flow is driven solely by the motion of the top plate. For case(b), it is found that significant reduction in entropy generation compared with the case of isothermal plates can be achieved by extracting heat through the moving plate at constant rate. If the moving plate is to supply heat to the fluid, then there is an optimum value of heat flux that minimizes the entropy generation. For cases (c) and (d) there exists optimum combinations of thermal parameters that result in minimum entropy generation. These optimum combinations have been identified. For the case of convection at both plates, i.e. case (d), the present analysis extends the work of Ibanez et al [6] by allowing the coolant temperature at the moving plate to be different from the coolant temperature at the stationary plate. For identical coolant temperatures as assumed by Ibanez et al [6], the present results validate their conclusions that minimum entropy generation can be realized with asymmetric convective cooling of the plates. 


\section{References}

1. Bejan, A., A study of entropy generation in fundamental convective heat transfer, ASME Journal of Heat Tansfer, 101, 718-725 (1979).

2. Bejan, A., Entropy Generation Minimization, CRC Press, New York, (1996).

3. Mahmud, S, and R. A.Frazer, The scond law analysis of fundamental convective heat transfer problems, International Journal of Thermal Sciences, 42,177-186 (2003).

4. Ebray, L.B., M.S.Ercan, B.Sulus, and M.M.Yalcin, Entropy generation during fluid flow between two parallel plates, Entropy,5, 506-518 (2003).

5. Ibanez, G., S. Cuevas, and M.L. de Haro, Minimization of entropy generation by asymmetric convective cooling,International Journal of Heat and Mass Transfer, 46,1321-1328 (2003).

6. Ibanez, G., S. Cuevas, and M.L. de Haro, Heat transfer in asymmetric convective cooling and optimized entropy generation rate, Revista Mexicana De Fisica, 49,338-343 (2003).

(C) 2006 by MDPI (htpp:/www.mdpi.org). Reproduction for noncommercial purposes permitted. 